\title{
Plasma Kallikrein as a Modulator of Liver Injury/Remodeling
}

Ibrahim A Ahmed ${ }^{1}$, Miran A Jaffa ${ }^{2}$, Mayssam Moussa ${ }^{1}$, Duaa Hatem ${ }^{1,3}$, Ghewa A El-Achkar ${ }^{1}$, Rola Al Sayegh ${ }^{1,4}$, Mia Karam ${ }^{1,5}$, Eva Hamade ${ }^{6}$, Aida Habib ${ }^{1,4 *}$ and Ayad A Jaffa ${ }^{1 *}$

${ }^{1}$ Department of Biochemistry and Molecular Genetics, Faculty of Medicine, Beirut, Lebanon, ${ }^{2}$ Epidemiology and Population Health Department, Faculty of Health Sciences, American University of Beirut, Beirut, Lebanon, ${ }^{3}$ Section of Pharmacology, Department of Bioethics and Safety, Catholic University, Rome, Italy, ${ }^{4}$ INSERM-UMR1149, Centre de Recherche sur I'Inflammation, and Sorbonne Paris Cité, Laboratoire d'Excellence Inflamex, Faculté de Médecine, Site Xavier Bichat, Universite de Paris, Paris, France, ${ }^{5}$ Biomedical Engineering Program, Maroun Semaan Faculty of Engineering and Architecture, American University of Beirut, Beirut, Lebanon, ${ }^{6}$ Laboratory of Cancer Biology and Molecular Immunology, Faculty of Sciences I, Lebanese University, Beirut, Lebanon

OPEN ACCESS

Edited by:

Emanuela Ricciotti,

University of Pennsylvania,

United States

Reviewed by:

Zia Shariat-Madar,

University of Mississippi, United States

Neil J. Grimsey,

University of Georgia, United States

*Correspondence: Aida Habib ah31@aub.edu.Ilb

Ayad A. Jaffa aj24@aub.edu.lb

Specialty section: This article was submitted to Inflammation Pharmacology, a section of the journal

Frontiers in Pharmacology

Received: 26 May 2021

Accepted: 23 July 2021 Published: 09 September 2021

Citation:

Ahmed IA, Jaffa MA, Moussa M, Hatem D, El-Achkar GA, Al Sayegh R, Karam M, Hamade E, Habib A and Jaffa AA (2021) Plasma Kallikrein as a Modulator of Liver Injury/Remodeling.

Front. Pharmacol. 12:715111. doi: 10.3389/fphar.2021.715111
The occurrence and persistence of hepatic injury which arises from cell death and inflammation result in liver disease. The processes that lead to liver injury progression and resolution are still not fully delineated. The plasma kallikrein-kinin system (PKKS) has been shown to play diverse functions in coagulation, tissue injury, and inflammation, but its role in liver injury has not been defined yet. In this study, we have characterized the role of the PKKS at various stages of liver injury in mice, as well as the direct effects of plasma kallikrein on human hepatocellular carcinoma cell line (HepG2). Histological, immunohistochemical, and gene expression analyses were utilized to assess cell injury on inflammatory and fibrotic factors. Acute liver injury triggered by carbon tetrachloride $\left(\mathrm{CCl}_{4}\right)$ injection resulted in significant upregulation of the plasma kallikrein gene (Klkb1) and was highly associated with the high mobility group box 1 gene, the marker of cell death $(r=0.75, p<0.0005, n=7)$. In addition, increased protein expression of plasma kallikrein was observed as clusters around necrotic areas. Plasma kallikrein treatment significantly increased the proliferation of $\mathrm{CCl}_{4}$-induced HepG2 cells and induced a significant increase in the gene expression of the thrombin receptor (protease activated receptor-1), interleukin 1 beta, and lectin-galactose binding soluble 3 (galectin-3) $(p<0.05, n=4)$. Temporal variations in the stages of liver fibrosis were associated with an increase in the mRNA levels of bradykinin receptors: beta 1 and 2 genes $(p<0.05 ; n=3-10)$. In conclusion, these findings indicate that plasma kallikrein may play diverse roles in liver injury, inflammation, and fibrosis, and suggest that plasma kallikrein may be a target for intervention in the states of liver injury.

Keywords: plasma kallikrein, necrosis, inflammation, liver injury, kallikrein-kinin system, fibrosis, remodeling

\section{INTRODUCTION}

Liver injury is an eminent condition of the body system owing to the numerous functions of the liver. Hepatitis (Real et al., 2019), acute liver failure (Stravitz and Lee, 2019), cholestasis (Horvatits et al., 2019), nonalcoholic fatty liver disease (Singh et al., 2015), and alcoholic liver disease (Gao and Bataller, 2011) are the varying phenotypes resulting from acute and chronic liver injuries. Some of the hallmarks of these pathologies reside in the initiation of cell death (Wang, 2014), inflammatory (Zhangdi et al., 2019), and fibrotic mechanisms (Weiskirchen et al., 2018). 
The coagulation system, a functional player of the cardiovascular system, is indicated as a driving force in liver injury and remodeling (Nault et al., 2016; Pant et al., 2018). This system modulates physiological and pathophysiological actions pertaining to neutrophil aggregation, vasodilation, inflammation, complement activation, and vascular tone (Ribeiro et al., 2014; Schmaier, 2016; Kenne et al., 2019). Previous studies of acute and chronic inflammation, and tissue remodeling have highlighted the role of the kallikrein-kinin system (KKS) (Ribeiro et al., 2014). The thrombin receptors, protease-activated receptors (PAR) 1 and 2 (Kallis et al., 2014; Nault et al., 2016; Shearer et al., 2016), and the bradykinin receptor, beta 2 (BDKRB2), are upregulated in liver fibrosis with a possible involvement of the latter and its ligand, bradykinin (BK) in fibrotic resolution (Sancho-Bru et al., 2007). However, other studies have shown that BDKRB2 is implicated in immune liver injury (Zhang et al., 2019), while the inhibition of the bradykinin receptor, beta 1 (BDKRB1), was shown to resolve the disorder (Zhang et al., 2018). Agonists of PARs like thrombin, tissue factor, trypsin, mast tryptase, coagulation factors Xa and VIIa, and plasma kallikrein have been studied over the past years (Gieseler et al., 2013; Heuberger and Schuepbach, 2019). Most compelling evidence stems from thrombin/factor Xa-induced PAR1 involvement in tissue fibrogenesis, while mast cell tryptase and factor $\mathrm{Xa}$ act through PAR2 to cause fibroblast proliferation, differentiation, and migration (Borensztajn et al., 2008; Borensztajn et al., 2010; Kitasato et al., 2014). In addition, experimental and human studies of acute and chronic liver disease showed the upregulation of thrombin, factor $\mathrm{Xa}$ and tissue factor, therefore implicating them as inducers of PARs in liver fibrogenesis and fibrosis progression (Marra et al., 1995; Pant et al., 2018). The cleavage of transforming growth factor, beta 1 , the most potent fibrogenic factor, was recently described by plasma kallikrein, in hepatic stellate cells, and suppressing this mechanism by inhibition prevented acute liver injury ( $\mathrm{Li}$ et al., 2018). This implicates plasma kallikrein as a driver of liver injury, yet more investigations are needed to ascertain this effect.

In this study, we explored the involvement of the PKKS in acute and chronic liver injury to unravel its possible roles in cell death, inflammation, and fibrosis. We applied correlation analysis to explore/establish relationships, risk, interactions, and possible involvement of some players as mediators of liver injury. Our findings demonstrated that components of the PKKS are associated with cell death, inflammation, myofibroblast activators, as well as fibrosis of liver injury, and may function as the indicators of oscillating molecular regulation in different phases of liver injury.

\section{MATERIALS AND METHODS}

\section{Animals Experimental Liver Injury}

C57BL/6J male mice of 10-12 weeks old were used throughout the experiments and were obtained from the animal facility of the American University of Beirut. The mice were housed five per cage in a temperature- and humidity-controlled room, kept on a 12-hr light-dark cycle, and provided with food and water ad lib.
All experimental procedures were approved and conducted following the guidelines of the Institutional Animal Care and Use Committee (IACUC: 19-08-541 and 19-08-542)

Acute liver injury: Acute liver injury was induced by a single intraperitoneal (i.p.) injection of $0.6 \mathrm{ml} / \mathrm{kg}$ carbon tetrachloride $\left(\mathrm{CCl}_{4}\right)$ (270652 Sigma-Aldrich) diluted $(1 / 10)$ in mineral oil (vehicle) (M5904 Sigma-Aldrich). The mice were sacrificed by cervical dislocation, and the liver tissues were harvested at each corresponding time point (Figure 1A).

Liver fibrosis: Chronic liver injury was induced by i.p. injection of diluted $(1 / 10) 0.6 \mathrm{ml} / \mathrm{kg} \mathrm{CCl}$ in mineral oil, twice a week for $2.5,4,6$, or 7 weeks. Mice were sacrificed at the 1 st or 3 rd day after the last injection of $\mathrm{CCl}_{4}$.

\section{Histological and Immunohistochemical Experiments}

Upon sacrifice, samples of each of the four liver lobes were cut and placed on microscopic slides. Cell death by necrosis was evaluated on hematoxylin and eosin (H\&E), and collagen fiber deposition was carried out by the picro sirius red (PSR) staining. Immunohistochemistry (IHC) for plasma kallikrein was performed using a rabbit polyclonal antibody anti-plasma kallikrein (PA5-76711 Invitrogen), biotinylated goat anti-rabbit secondary antibodies were added for an hour while staining using 3,3'-diaminobenzidine (DAB), and counterstaining with hematoxylin (Leica Biosystem) was carried out as described previously (Habib et al., 2019). No staining was observed when omitting the primary antibody. Evaluation and quantification of necrosis and collagen fiber deposition were performed on photomicrographs of ten each per mouse, using ImageJ software (NIH, United States). Necrotic areas, identified by the absence of, or altered hepatic cells, were delineated and calculated to the total area of each photomicrograph. Collagen fibers were identified by their red deposition and quantified by utilizing ImageJ software.

\section{Hepatocellular Carcinoma Cell Line, Culture, and Treatment}

Human hepatocellular carcinoma cell line, HepG2, was obtained from the ATCC (Virginia, United States). All necessary procedures from storage to culturing were performed according to the guidelines of the ATCC. The cells were cultured in low-glucose Dulbecco's media (DMEM, D6046 Sigma-Aldrich) supplemented with $10 \%$ fetal bovine serum (FBS) and $1 \%$ penicillin-streptomycin (P/S), and incubated at $37^{\circ} \mathrm{C}$. 1 or $2 \mathrm{mM} \mathrm{CCl}_{4}$ in $0.5 \%$ dimethyl sulfoxide (DMSO, 41640 Sigma-Aldrich), or $2.5 \mathrm{ng} / \mathrm{ml}$ plasma kallikrein (K2638 SigmaAldrich) were added to the cells. Viability and proliferation assays were conducted using a 96-well plate. HepG2 cells were plated in $1 \times 10^{5}$ cells/well for viability assay (for cell death or toxicity determination) and $1 \times 10^{4}$ cells/well for the proliferation assay. After the treatment of cells with $\mathrm{CCl}_{4}$ or/and plasma kallikrein for $24 \mathrm{~h}$, viability and proliferation tests were measured using the MTT assay (M5655 Sigma-Aldrich) at an absorbance of $595 \mathrm{~nm}$, and calculated according to the instruction of the manufacturer. 


\section{A}

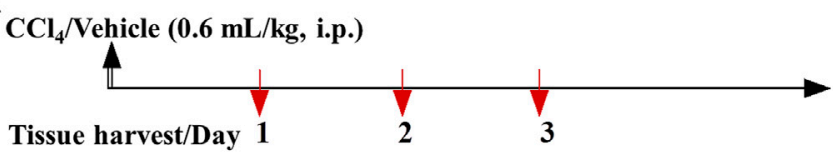

B
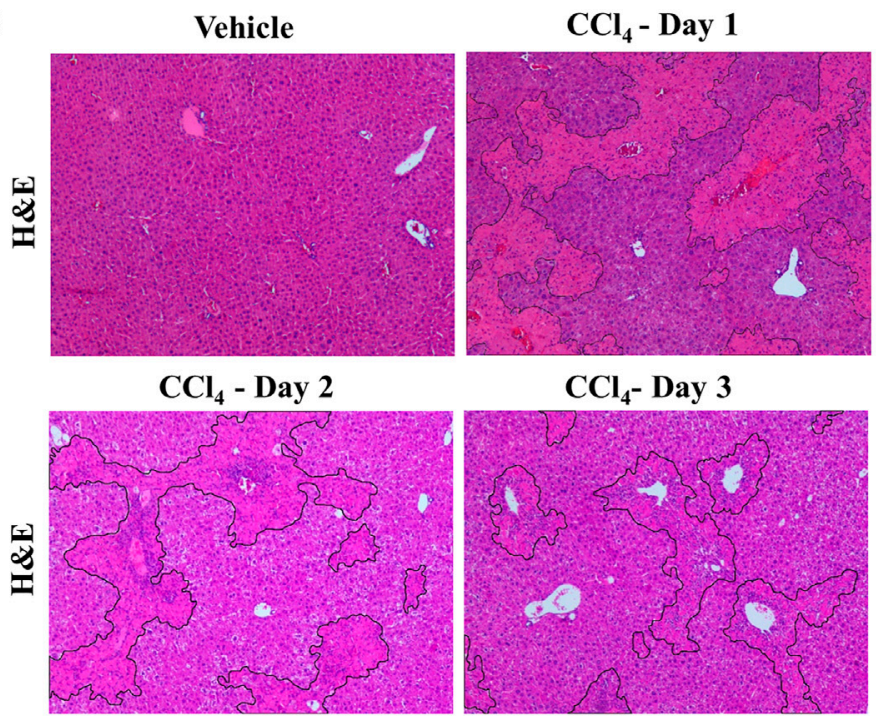

\section{Hepatic Necrosis}

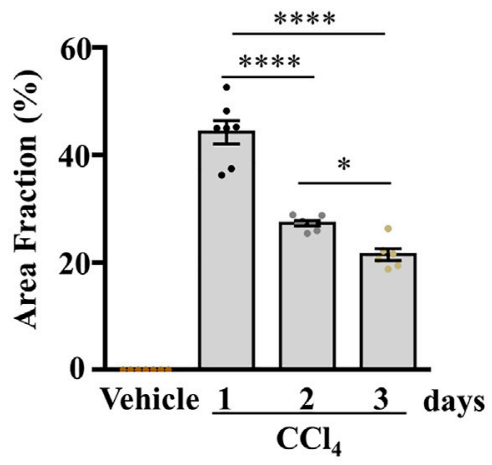

C

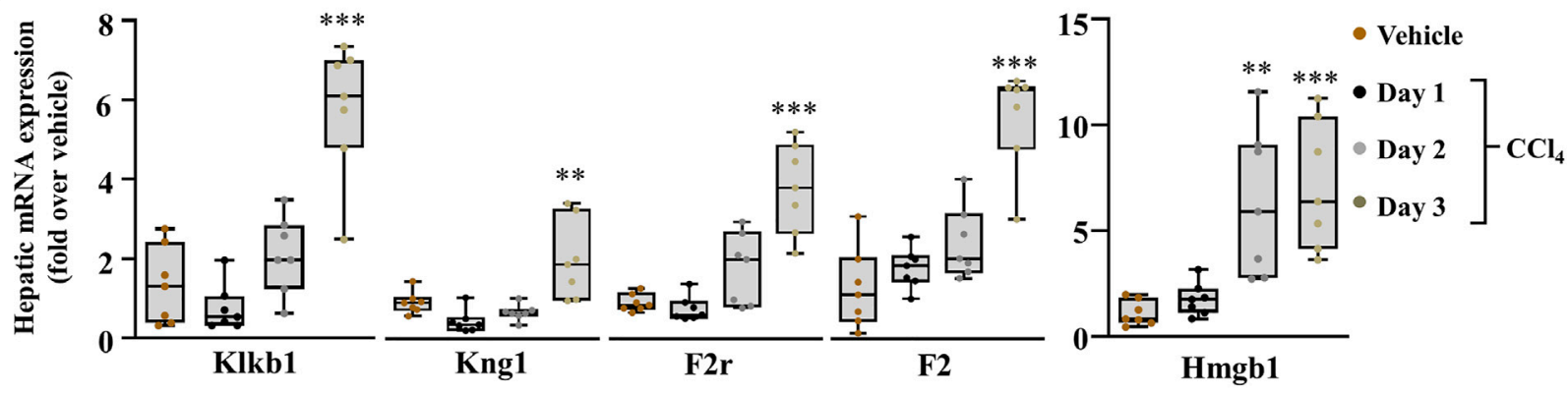

D
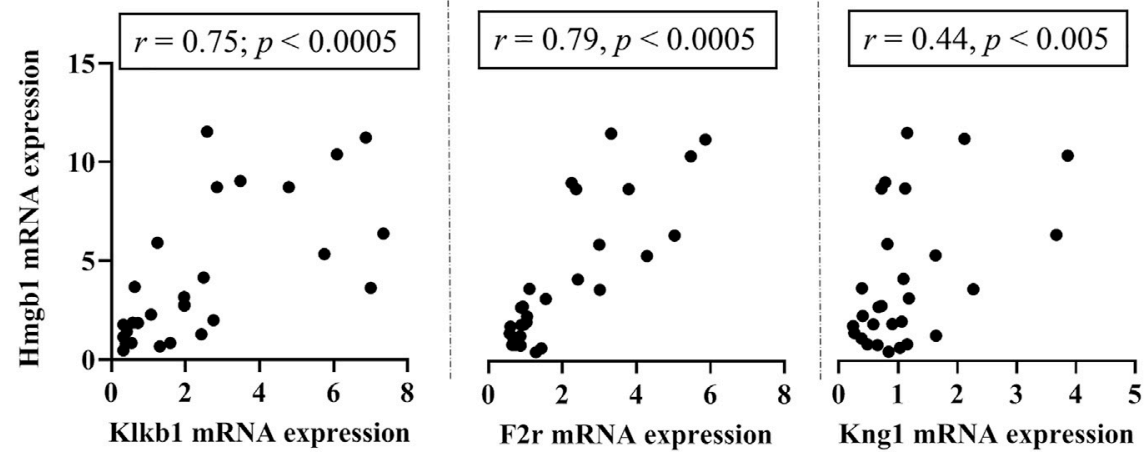

FIGURE 1 | Pronounced modification in the hepatic PKKS genes in acutely injured mice. (A) Schematic representation of carbon tetrachloride (CCl 4 )-induced acute liver injury at days 1, 2, and 3 after the $\mathrm{CCl}_{4}$ and mineral oil (vehicle) injection. (B) Representative H\& $\mathrm{E}$ (original magnification $\times 40$ ) staining of liver sections; necrotic areas are delineated by marked areas quantified using ImageJ software; data are represented as mean \pm SEM (one-way ANOVA followed by Sidak's multiple comparisons, ${ }^{\star} p<0.05,{ }^{\star \star \star \star} p<0.0001 ; n=7$ mice per group). (C) Gene expression analysis of the PKKS (Klkb1, Kng1, F2r, and F2) and Hmgb1 genes. Data are shown as minimum to maximum values of box plots with whiskers extending 1.5 times the interquartile range. Center lines indicate the medians, while box limits represent the 25 th and 75 th percentiles $\left(n=7\right.$ per group). Statistical significance was determined by one-way ANOVA followed by Sidak's multiple comparisons, where ${ }^{\star \star} p<0.005$, ${ }^{* \star *} p<0.0005$ ( $\mathrm{CCl}_{4}$ vs. Vehicle). (D) Association between Hmgb1 and Klkb1, F2r or Kng1 genes, correlative plots were assessed by the Spearman correlation. 
$3 \times 10^{5}$ HepG 2 cells/well were plated in a 12 -well plate for the plasma kallikrein study. RNA extraction and gene expression analysis were performed after the cells were treated with $2.5 \mathrm{ng} /$ $\mathrm{ml}$ plasma kallikrein or media only (control groups) for $24 \mathrm{~h}$.

\section{Gene Expression Profiles}

Total RNA from the frozen liver tissue fragments obtained from the left and median lobes, homogenized in Qiazol Lysis Reagent (79306 Qiagen, Hilden, Germany), using a Tissue Lyser (QIAGEN II), was extracted as described previously (Habib et al., 2019). For HepG2 cells, the total RNA was extracted using TRIzol ${ }^{\mathrm{TM}}$ Reagent (15596026 Ambion Life Technologies). $2 \mu \mathrm{g}$ of the total RNA were reverse-transcribed into cDNA using the High-Capacity Reverse Transcriptase kit (004007363 Thermo Fisher Scientific). Using the iTaq $^{\text {TM }}$ Universal SYBR Green Supermix (1725121 Bio-Rad Laboratories), real-time quantitative polymerase chain reactions (RT-qPCR) were performed in a CFX384 system (Bio-Rad Laboratories, California, United States). The primers (Macrogen Inc., Seoul, South Korea) were previously described (Habib et al., 2019), and others are listed in Supplementary Table 1. The results were calculated using the $\Delta \Delta C T$ method and normalized in the housekeeping genes $18 \mathrm{~S}$ and GAPDH for liver tissues and HepG2 cells, respectively.

\section{Statistical Analysis}

Statistical analysis was conducted using SPSS (Statistical Package for the Social Sciences) software and GraphPad Prism 8, (version 8.4.3 for Windows, GraphPad Software, La Jolla, CA 92037, United States). The test of normality was performed using the Shapiro test, while multiple comparisons between groups were conducted by one-way analysis of variances (ANOVA) followed by Sidak's multiple comparison test or the Mann-Whitney U test; ${ }^{*} p<0.05,{ }^{* *} p<0.005,{ }^{* * *} p<0.0005$, and ${ }^{* * * *} p<0.0001$ are considered statistically significant. Correlation analysis was carried out by the Spearman correlation of nonparametric test, while the significance of the coefficient of correlation, $r$, was determined by $p<0.05$.

\section{RESULTS}

\section{Genes for Plasma Kallikrein (KIkb1), High Molecular Weight Kininogen (Kng1), and PAR1 (F2r) Are Positively Associated to the Gene of High Mobility Group Box (Hmgb1), in Acute Liver Injury}

We studied the role of the PKKS in acute liver injury of C57BL/6J mice treated with a single injection of $0.6 \mathrm{ml} / \mathrm{kg}$ of $\mathrm{CCl}_{4}$ i.p. Mice were sacrificed at day one, two, or three post $\mathrm{CCl}_{4}$ injection (Figure 1A). Necrosis was assessed by H\&E staining on liver sections (Figure 1B delineated area) and showed a $45.1 \%$ increase in necrosis after day one of $\mathrm{CCl}_{4}$ treatment compared to the vehicle. A gradual decrease in this necrotic area was observed from days one to two, and two to three (39.3 and 22.9\%, respectively, Figure 1B). Compared to vehicle-treated animals, injured liver presented a $42.5 \%$ decrease in hepatic mRNA expression of the Klkb1 gene at day one, and a 1.6- and 4.3-fold increase at days two and three, respectively (Figure 1C; $p<0.0005$ at day three). Since plasma kallikrein activation modulates a high molecular weight kininogen (KNG1) (Moreau et al., 2005) and PAR1 signaling (Abdallah et al., 2010), we assessed their gene expression. Figure 1C shows a similar pattern of expression of 51.9 and $16.7 \%$ decrease 1 day after the last $\mathrm{CCl}_{4}$ injection in the hepatic mRNA levels of Kng1 and F2r. This suggests a modulation in the downstream target of plasma kallikrein. Since PAR1 has numerous ligands including plasma kallikrein, we assessed the thrombin gene (F2) expression in the liver, which showed an increased expression at day one injury, in contrast to the decrease depicted for Klkb1, Kngl, and F2r genes (Figure 1C). Next, we assessed the gene expression of high mobility group box (Hmgb1), a marker of cell death, which has been shown to have pro-inflammatory effects (Man et al., 2015; Paudel et al., 2018; Zhang et al., 2019). We observed a similar pattern of the expression of the Hmgbl gene to the induced PKKS genes (Figure 1C). The Spearman correlation analysis showed a positive association between Hmgbl gene and the PKKS genes (Klkbl, $r=$ $0.75 p<0.0005$; F2r, $r=0.79 ; p<0.0005$; Kng1, $r=0.44, p=0.019$; Figure 1D, Supplementary Figures 1A,B).

\section{In Vivo and In Vitro Impacts of Plasma Kallikrein in Liver Injury}

We further analyzed the expression of the plasma kallikrein protein by immunohistochemistry which showed a decrease on day 1 compared to the vehicle and formed clusters around the injured areas on day 2 and day 3 (Figure 2A; black arrows). To further investigate the role of plasma kallikrein in liver injury, we evaluated the effect of plasma kallikrein on the viability and proliferation of HepG2 cells in vitro. The effect of plasma kallikrein was tested and compared between $\mathrm{CCl}_{4}$-treated and non- $\mathrm{CCl}_{4}$-treated cells. First, HepG2 cells treated with 1 or $2 \mathrm{mM}$ of $\mathrm{CCl}_{4}$ for $24 \mathrm{~h}$ showed 40.8 and $60.5 \%$ cell death, respectively, whereas $2.5 \mathrm{ng} / \mathrm{ml}$ of plasma kallikrein showed no toxicity even at day 2 (Figures 2B,C). Likewise, plasma kallikrein increased the proliferation rate of HepG 2 cells by 20.4 and $72.6 \%$ at day 1 and day 2 of treatment, respectively, (Figure 2C). In $1 \mathrm{mM}$ $\mathrm{CCl}_{4}$-treated cells, plasma kallikrein elicited a $45.3 \%$ significant increase in proliferation compared to the $1 \mathrm{mM} \mathrm{CCl}_{4}$-treated cells alone $\left(p<0.0001\right.$, Figure 2D). Incubation of $2 \mathrm{mM} \mathrm{CCl}_{4}$-treated HepG2 cells with plasma kallikrein did not cause an increase in proliferation (Figure 2D), possibly due to the high toxicity of $2 \mathrm{mM} \mathrm{CCl}_{4}$ on the HepG2 cells. These results suggest a role in the proliferation of normal and necrosis-affected cells either through normally or necrosis-released molecular patterns. In parallel, we determined the effect of plasma kallikrein on HepG2 and observed a statistically significant induction of interleukin 1 beta (IL1B) and F2R genes but not PAR2 (F2RL1) gene expression (Figure 2D).

\section{The Induction of PKKS Genes Is Highly Associated to Inflammation and Immune Cell Recruiting Genes in the Liver}

The effectors of PKKS, plasma kallikrein, and bradykinin on their receptors have been implicated in vascular and tissue inflammation. Figure $\mathbf{3 A}$ shows an increase in the mRNA 


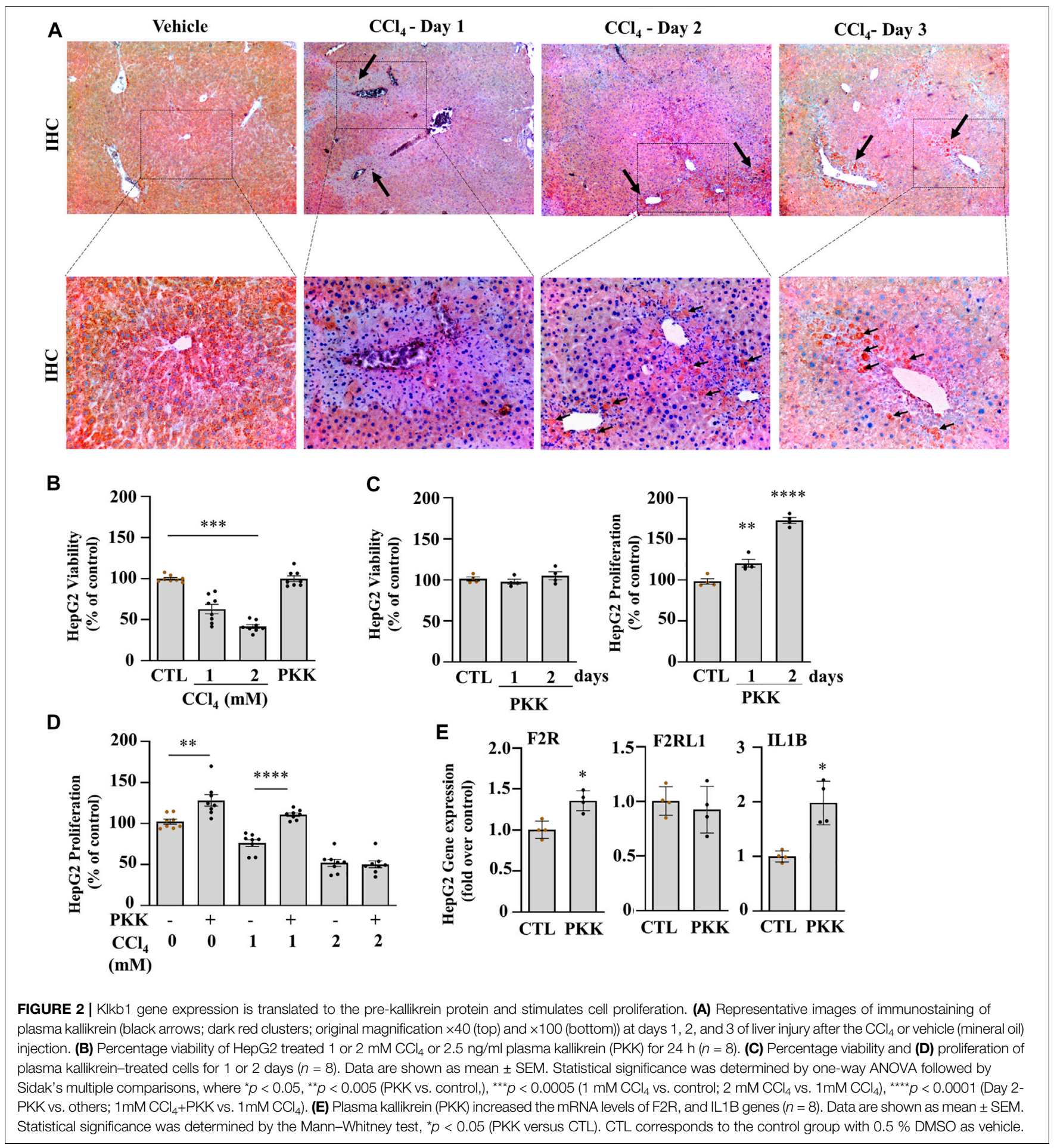

levels of the inflammatory players, tumor necrosis factor-alpha (Tnfa) and Interleukin $1 \mathrm{~b}$ (Illb) genes in the liver, starting at day 1 after $\mathrm{CCl}_{4}$ injection, and with a maximal expression at day 2 for Tnfa, and day 3 for Illb. Interleukin-6 (Il16) gene was not induced at the observed time point of acute liver injury. Spearman correlation studies with the PKKS and thrombin genes (F2) indicate a strong relationship with the Illb gene
(Figure 3B, Supplementary Figures 1A,B). Moreover, the expression of chemokine (C-C motif 2 and 3) $\mathrm{Ccl} 2$ and $\mathrm{Ccl} 3$ genes that may trigger the recruitment of immune cells to the site of injury (Mossanen et al., 2016; Reichel et al., 2012) was analyzed. Figure $4 \mathbf{A}$ indicates an increase in $\mathrm{Ccl} 2$ - and $\mathrm{Ccl} 3-\mathrm{mRNA}$ expressions, which peaked at day 2 for $\mathrm{Ccl} 2$ and day 3 for $\mathrm{Ccl} 3$. Ccl3 was strongly associated to the PKKS genes as 

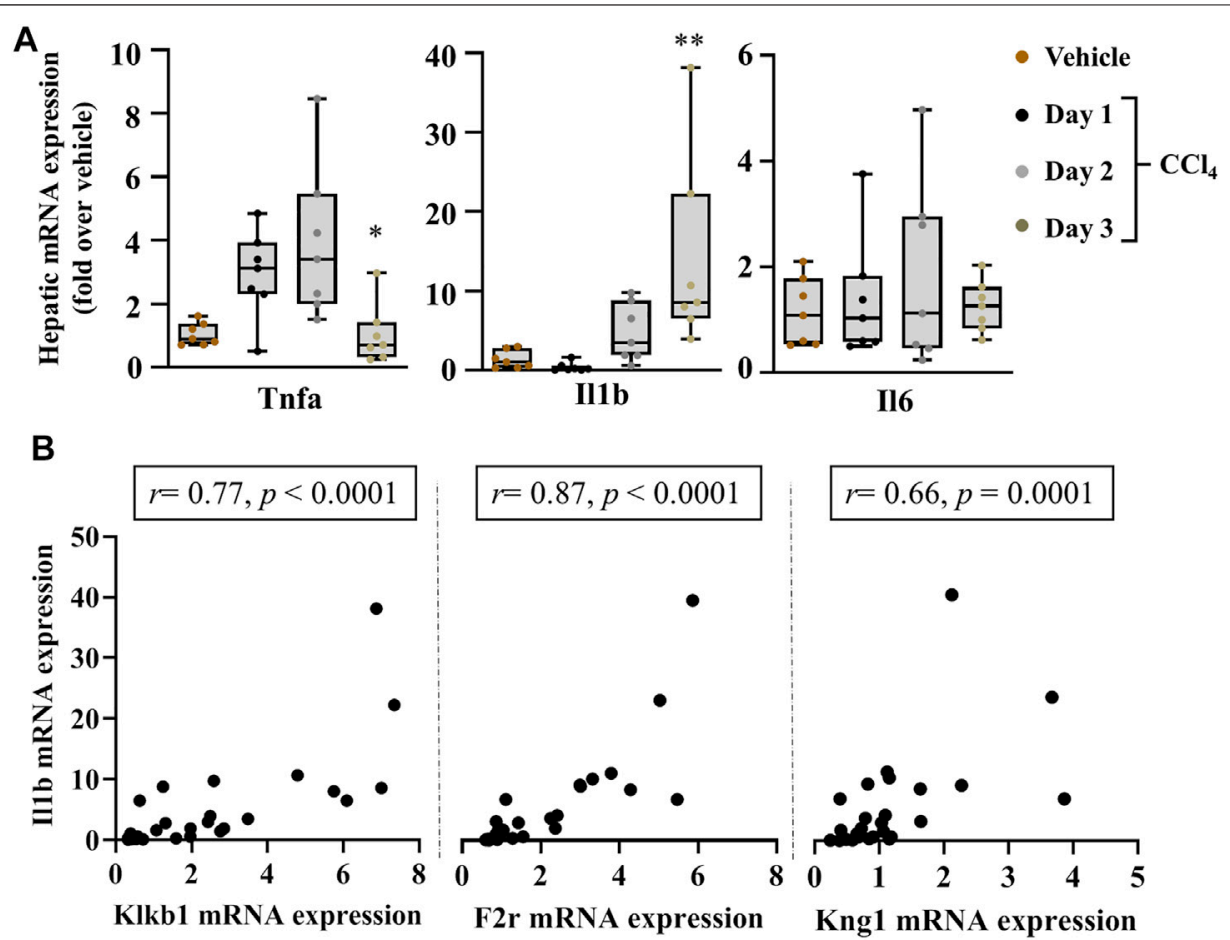

FIGURE 3| Strong association of PKKS genes to hepatic mRNA levels of inflammatory markers in acute liver injury. (A) Tnfa, II1b, and II6 gene induction implicates an inflammatory response to liver injury over the time course observed. (B) Spearman correlation studies between the Klklb1 gene and llb gene, the F2r gene and II1b gene, and the Kng1 gene and II1b gene. Data representation and statistical analysis were performed as described in the legend for Figure $1 \mathbf{C}\left(n=7\right.$ per group). ${ }^{\star} p<0.05$, ${ }^{\star \star} p<0.005\left(\mathrm{CCl}_{4}\right.$ vs. Vehicle), and correlative plots were assessed by the Spearman correlation. Vehicle corresponds to mineral oil.

observed by the correlation coefficient $(r=0.67,0.57$, and 0.77 , for Klkb1, Kng1, and F2r, respectively) (Figure $4 \mathbf{B}$, Supplementary Figures 1A,B). Different subsets of immune cells have been detected at the acute injury site. Genes for the adhesion $G$ protein-coupled receptor E1 (Adgre1), a marker of macrophage, lymphocyte antigen 6 complex, locus $\mathrm{C} 1$ and $\mathrm{G}$, Ly6c, and Ly6g (markers of monocyte and neutrophil presence) have been shown to increase their expression in acute liver injury and inflammation (Mitchell et al., 2009; Endig et al., 2019). We assessed the possibility of the PKKS gene association to these immune cell recruiting genes. The mRNA expression levels of Adgre1, Ly6c, and Ly6g were increased post $\mathrm{CCl}_{4}$ with a transient increase for the Ly6g and a sustained increase for Adgre1 and Ly6c (Figure 4A). The Adgre1 and Ly6c genes showed a strong association to the PKKS genes (Figure 4B, Supplementary Figures 1A,B). Although the Ly6g gene showed no association to the PKKS genes, (Supplementary Figures 1A,B), the expression of myeloperoxidase gene, Mpo, a marker of neutrophil activation, gradually increased (Figure 4A), and a strong positive correlation with the PKKS genes (Figure $4 \mathbf{B}$, Supplementary Figures 1A,B) was observed.

\section{The Induction of PKKS Genes}

Plasma kallikrein induces an upregulation of the gene for galectin-3 (lectin, galactose binding, soluble 3, and LGALS3) in HepG2 cells.
We analyzed the gene expression of myofibroblast activators, Lgals3 and Ccn2, the gene for connective tissue growyh factor (CTGF), and observed their increase in the mRNA level at day 1 after acute liver injury and a gradual decrease at day 2 till 3 in C57Bl/6J mice (Figure 5A). These genes showed no association with the PKKS genes (Figure 5B, Supplementary Figures 2A,B). Incubation of HepG2 cells with plasma kallikrein increased LGALS3 and decreased CCN2 gene expressions (Figure 5C), suggesting the involvement of plasma kallikrein in the modulation of these two myofibroblast activators in fibrosis.

\section{Fibrogenesis and Inflammation in Early Chronic Liver Injury: Regulation of Induced PKKS Genes}

To investigate the role of plasma kallikrein in chronic liver injury, liver fibrosis was induced in $\mathrm{C} 57 \mathrm{BL} / 6 \mathrm{~J}$ mice after subjection to $0.6 \mathrm{ml} / \mathrm{kg}$ of $\mathrm{CCl}_{4}$ for 2.5 weeks (Figure 6A). Exposure of the mice to $\mathrm{CCl}_{4}$ showed an increase in picro sirius red staining (Supplementary Figure 3A). In this setting, we analyzed the gene expression of hepatic Lgals3, Ccn2, and extracellular matrix remodeling players. Figure 6B showed a statistically significant increase in the mRNA expression of Lgals3, tissue inhibitor of metalloproteinase 1 (Timp1), and matrix metallopeptidase 2 (Mmp2) genes. Hepatic injury was assessed after the last $\mathrm{CCl}_{4}$ injection by $\mathrm{H} \& \mathrm{E}$ staining (Supplementary Figure 2B, left; delineated area) and showed a $29.4 \%$ increase in the necrotic 


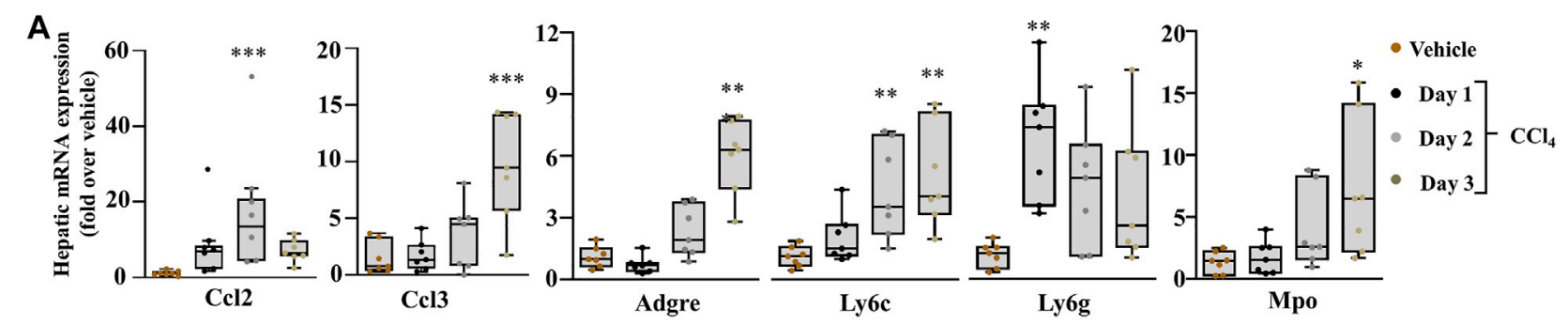

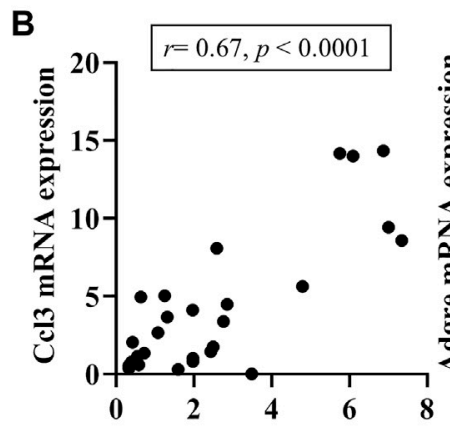

Klkb1 mRNA expression

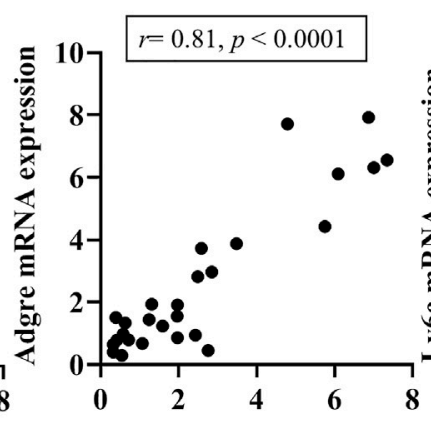

KIkb1 mRNA expression

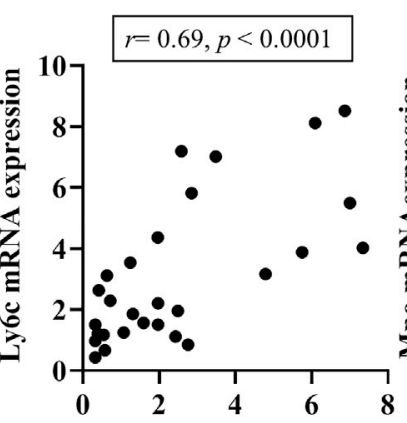

Klkb1 mRNA expression

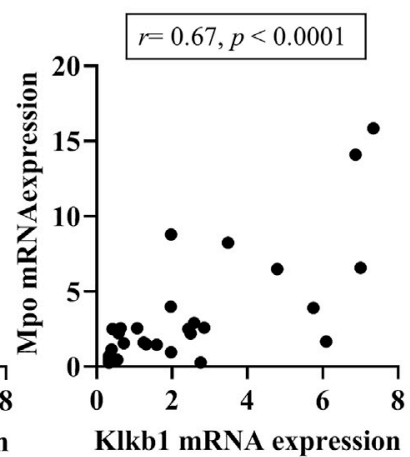

FIGURE 4 | Strong association of immune cells recruiting markers in acute liver injury. (A) Chemokine genes, Ccl2 and Ccl3; recruitment genes Adgre1, Ly6c, and Ly6g; and Mpo gene, a marker of neutrophil activation. (B) The correlation analysis by Spearman among the Klkb1 and Ccl3 genes; the Klkb1 and Adgre1 genes; the Klkb1 and Ly6c genes; and the Klkb1 and Mpo genes. Data representation and statistical analysis were performed as described in the legend for Figure 1C. Outliers are shown by dots outside the whiskers ( $n=7$ per group). ${ }^{\star} p<0.05,{ }^{\star \star} p<0.005,{ }^{\star \star \star} p<0.0005\left(\mathrm{CCl}_{4}\right.$ vs. Vehicle), and correlative plots were assessed by Spearman correlation. Vehicle corresponds to mineral oil.

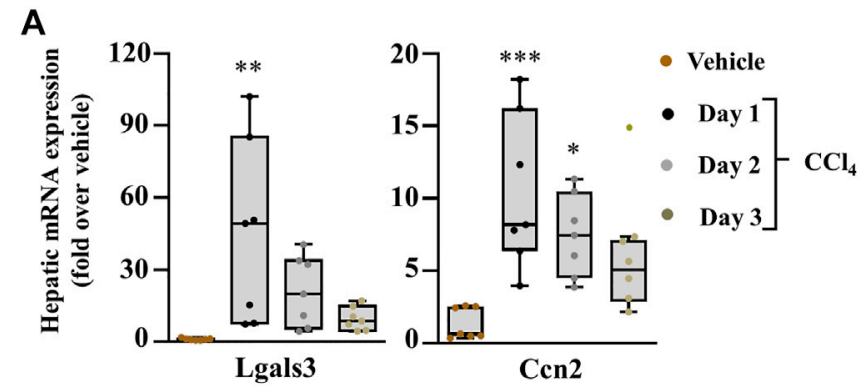

C

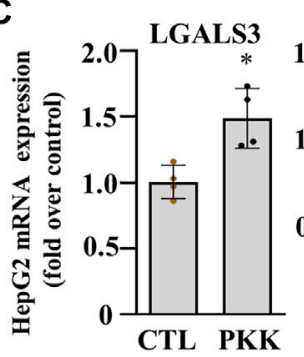

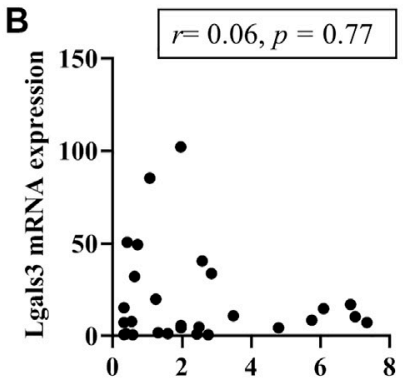

Klkb1 mRNA expression

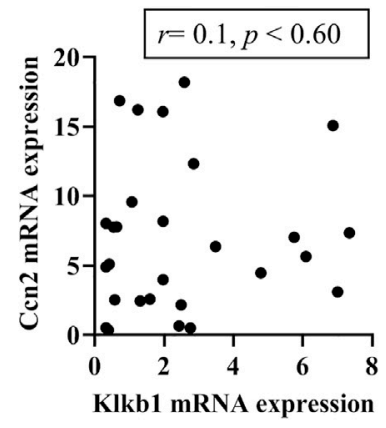

FIGURE 5 | Early induction of myofibroblast markers of activation in acute liver injury. (A) mRNA expression of Lgals3 and Ccn2. Data representation and statistical analysis were performed as described in the legend for Figure 1C. $\left(n=7\right.$ per group). Outliers are shown by dots outside the whiskers, ${ }^{\star} p<0.05^{\star \star} p<0.005,{ }^{\star \star \star} p<0.0005$ ( $\mathrm{CCl}_{4}$ vs. Vehicle). (B) Association of Klkb1, and Lgals3 and Ccn2, correlative plots were assessed by Spearman correlation. Vehicle corresponds to mineral oil. (C) Gene expression of LGALS3 and CCN2 in HepG2 cells were induced with plasma kallikrein (PKK). Data are shown as mean \pm SEM ( $n=4$ per group), and statistical significance was determined by the Mann-Whitney test; ${ }^{\star} p<0.05$ (PKK vs. CTL). CTL corresponds to culture media only. 
A

A last $\mathrm{CCl}_{4}$ injection

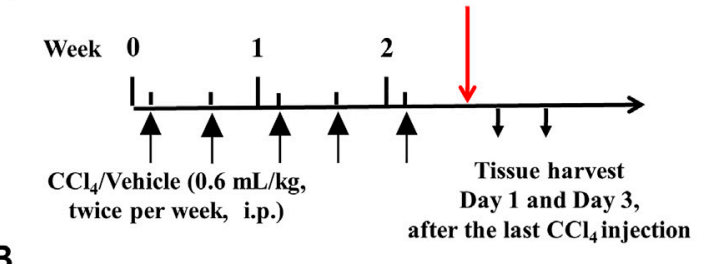

B
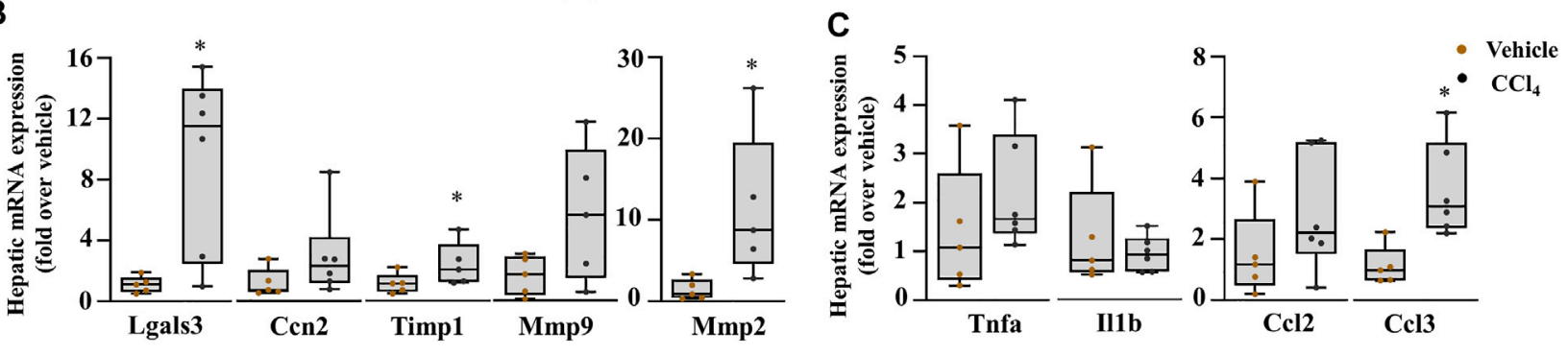

D

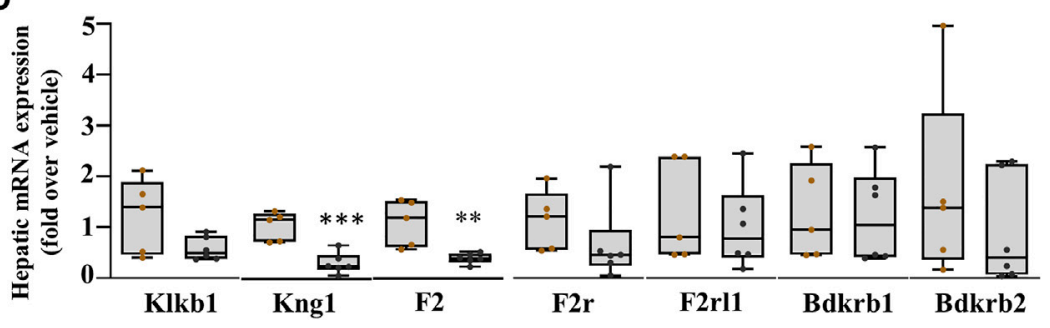

FIGURE 6 | Upregulation of markers of fibrosis: inflammatory processes are regulated. (A) Schematic representation of $\mathrm{CCl}_{4}$-induced chronic liver injury. Hepatic mRNA expression of (B) Lgals3, Ccn2, Timp1, Mmp9, and Mmp2; (C) inflammatory and chemokine genes, Tnfa, II1b, Ccl2, and Ccl3; (D) the PKKS genes and thrombin (F2). Data representation for box plots was performed as described in the legend for Figure 1C ( $n=5-6$ mice per group); statistical significance was determined by the Mann-Whitney test, where ${ }^{*} p<0.05,{ }^{* *} p<0.005$ ( $\mathrm{CCl}_{4}$ vs. Vehicle). Vehicle corresponds to mineral oil.

area when compared to vehicle (Supplementary Figure 2B). The mRNA level of Hmgb1 was unchanged in this time point of liver fibrosis (Supplementary Figure 2C). Assessment of chemokine genes showed a statistically significant increase for the $\mathrm{Ccl} 3$ gene (Figure 6C). Ccl2, Tnfa, and Illb remained unchanged (Figure 6C). Likewise, the Mpo gene revealed an unchanged gene expression compared to vehicle-treated animals (Supplementary Figure 2C). These results suggest the regulation of some inflammatory processes and fibrogenesis, contributing to ECM deposition. This is also observed in the PKKS genes where some genes were significantly downregulated (Kng1 and F2), and others with a tendency to decrease (Klkb1 and F2r) and unchanged (F2rl1, Bdkrb1, and Bdkrb2) (Figure 6D).

\section{Correlation Analysis of Klkb1 and Kng1 Gene Expression Suggests Different Roles at Different Time Points of Liver Fibrosis}

To corroborate the results of liver fibrogenesis on the PKKS effectors (plasma kallikrein and KNG1) and thrombin in chronic liver injury, we studied different time points of $\mathrm{CCl}_{4}$-induced liver fibrosis (4, 6, and 7 weeks). We analyzed their association to the myofibroblast activation markers, and fibrotic areas at all time points of chronic liver injury, including 2.5 weeks. The mice were treated with the same volume of vehicle or $\mathrm{CCl}_{4}$ twice per week and sacrificed after the last injection (Figure 7A; similar treatment pattern was performed at the 6th and 7 th weeks). Fibrosis was established at all time points (Figure 7B). There was a gradual increase in sirius red staining from approximately 3.0 folds in the 4 -week time point to 3.4 and 4.2 folds in the 6- and 7 -week time points, respectively (Figure 7C). The genes of myofibroblast activation markers, Lgals3 and $\mathrm{Ccn} 2$, were increased at all time points (Figures 7D,E). Klkb1 gene decreased at 6- and 7-week time points (Figure 7F). Supplementary Figures 3A-D showed an insignificant weak relationship between the Klkb1 mRNA expression to fibrotic area or gene expressions of Lgals3 and Ccn2 across all time points, except for the fibrotic area at 6 weeks $(r=0.71, p=0.05$, Figure 7G). We hypothesized a similar pattern of gene expression of Kng1 and Klkb1 mRNA levels due to their complex formation in the plasma and stoichiometric interaction (Kusumam et al., 2009; Kaplan and Ghebrehiwet, 2010). However, the Kng1 gene (Figure 7H) displayed a significant upregulation across all $\mathrm{CCl}_{4}$ time points except at the 6-week time point. Correlation analysis of the Kng1 gene showed a strong relationship at all time points, except at the 6week time point, suggesting a more involved and different role compared to the Klkb1 gene (Figure 7I, Supplementary Figures 3A-D, 4A-D). We showed that plasma kallikrein modulates $\mathrm{Ccn} 2$ gene expression (Figure 5C) and differs from thrombin in 
A last $\mathrm{CCl}_{4}$ injection

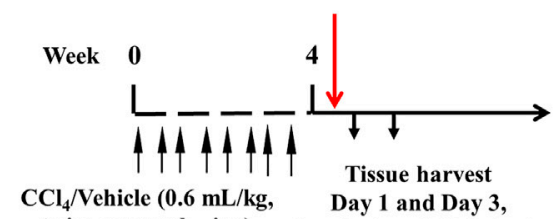

twice per week, i.p.) after the last $\mathrm{CCl}_{4}$ injection

B
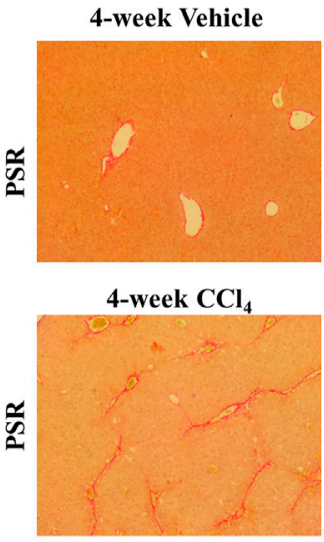

6-week Vehicle

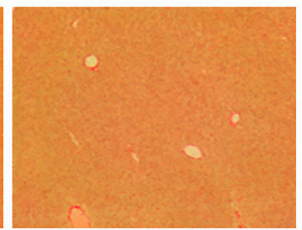

6-week $\mathrm{CCl}_{4}$

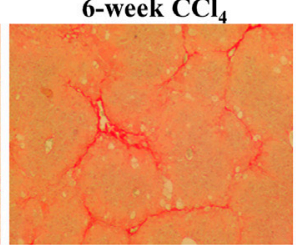

7-week Vehicle

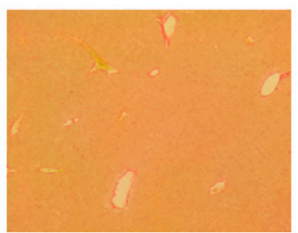

7-week $\mathrm{CCl}_{4}$

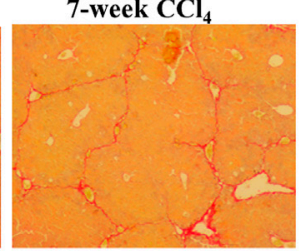

C

Picro Sirius Red

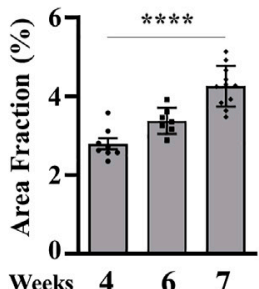

D

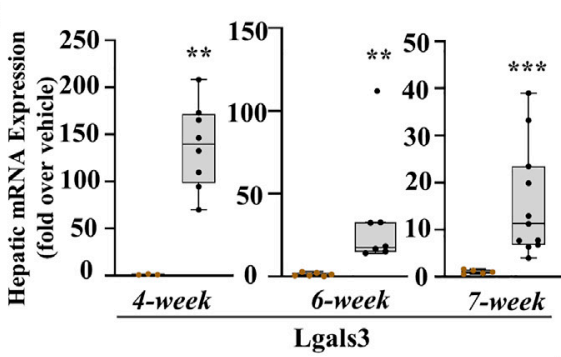

G

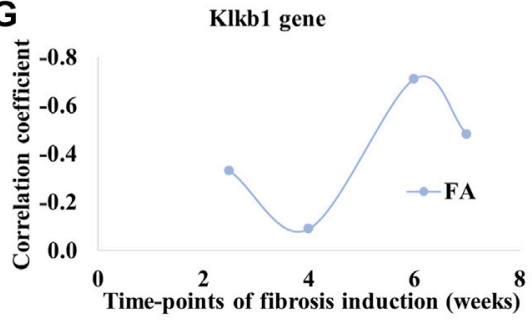

J

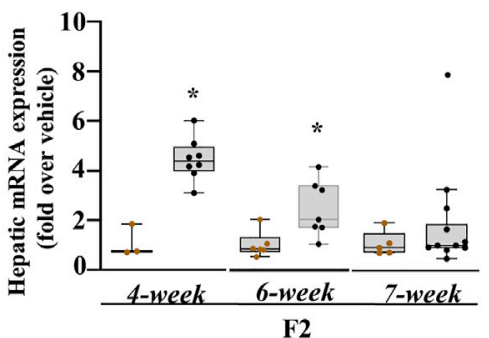

$E$
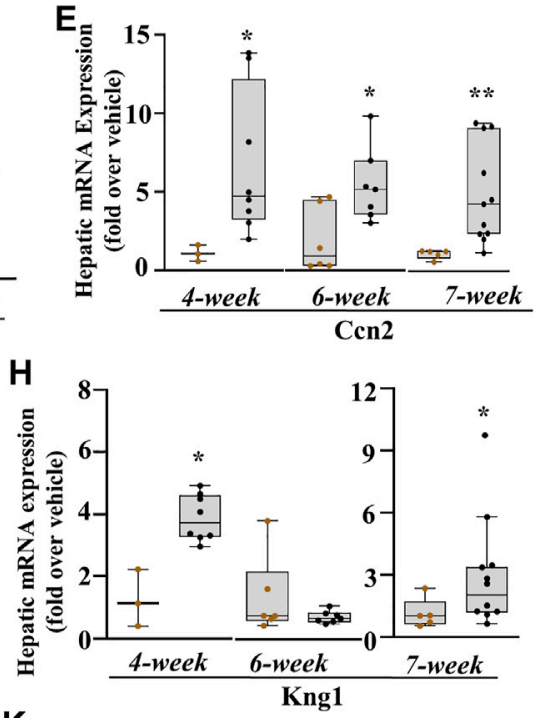

$\mathrm{K}$

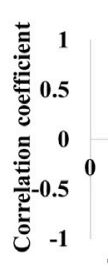

F2 gene

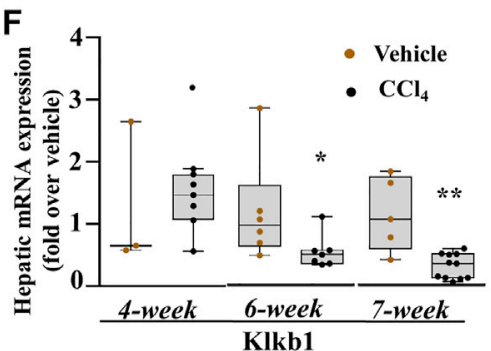

I Kng1 gene

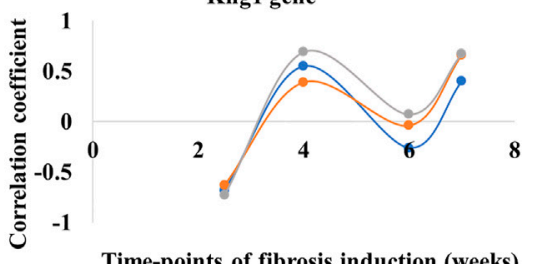

Time-points of fibrosis induction (weeks)

$\rightarrow$ FA $\rightarrow$ Ccn2 $\rightarrow$ Lgals 3

FIGURE 7 |KIkb1 and Kng1 gene expressions at different time points of the chronic liver injury model. (A) Schematic representation of CCl 4 -induced liver fibrosis at the 4 th week. Similar pattern of mice injections was performed at the 6th and 7th weeks as well. (B) Varying representative images of collagen fibers stained with picro sirius red (stained red). (C) Quantification of histological staining by ImageJ software (data representation by mean \pm SEM, $n=3-10$ mice per group: one-way ANOVA followed by Sidak's multiple comparisons; $\left.{ }^{\star \star \star \star} p<0.0001\right)$. The quantified areas in each sample were normalized to their average time points of controls before statistical analysis. Hepatic mRNA expression (D, E, F) of Lgals3, Ccn2, and Klkb1; (G) plot analysis of Klkb1 gene and (H) Kng1 gene; (I) plot analysis of Kng1 gene and (J) F2 gene; (K) plot analysis of F2 gene. Data representation for box plots was performed as described in the legend for Figure 1C. Outliers are shown by dots outside the whiskers ( $n=3-6$ mice for Vehicle and 7-10 for $\mathrm{CCl}_{4}$ treatment groups); statistical significance was determined by the Mann-Whitney test, where ${ }^{*} p<0.05$, ${ }^{* *} p<$ $0.005,{ }^{* \star *} p<0.0005,{ }^{* \star \star *} p<0.0001$ ( $\mathrm{CCl}_{4}$ vs. Vehicle). Vehicle corresponds to mineral oil. 


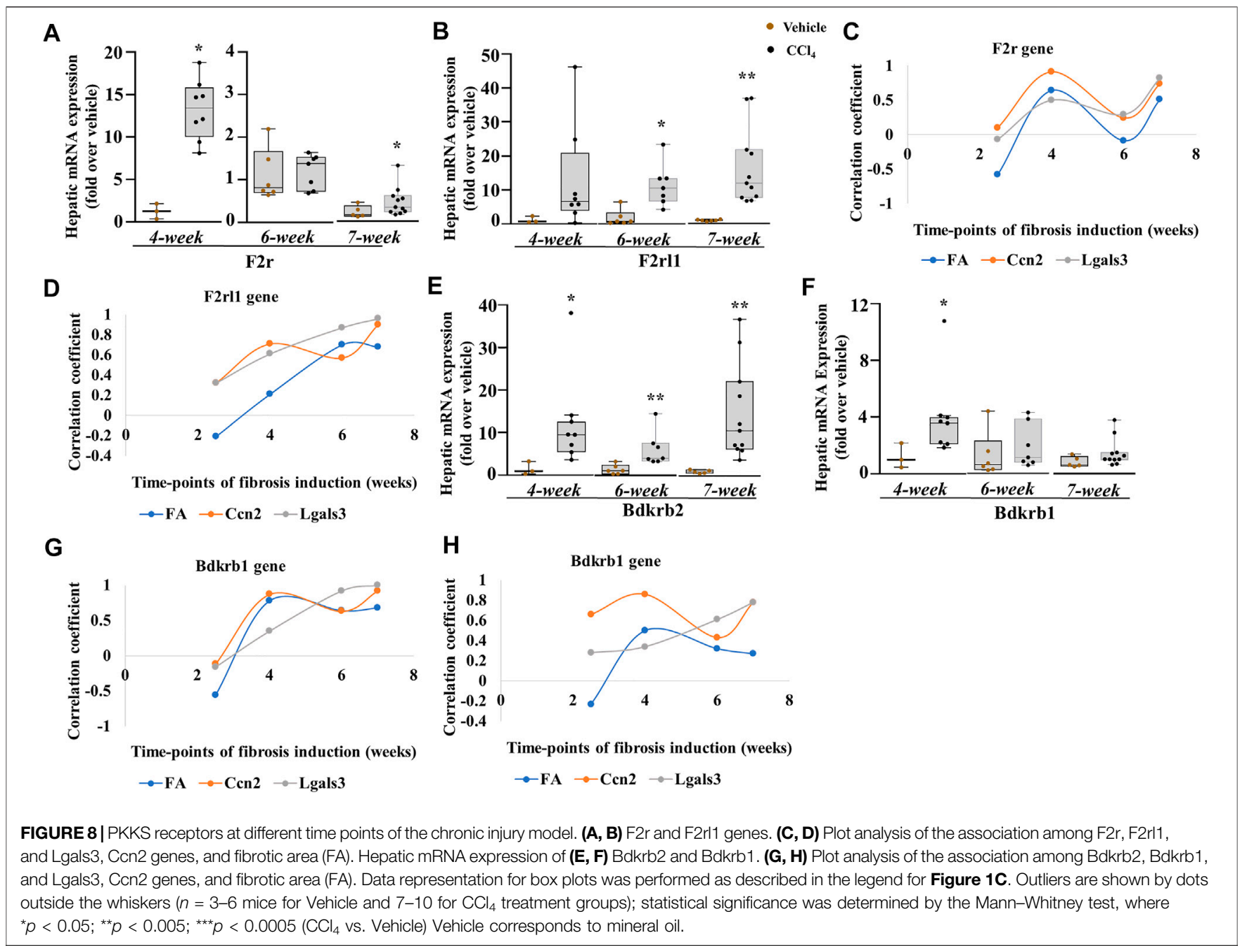

acute liver injury. In the observed time points, the gene expression of thrombin (F2) gene (Figure 7J) increased at the 4th and 6th weeks and formed a more robust correlative relationship compared to the Klkb1 gene expression (Figure $7 \mathbf{K}$, Supplementary Figures 3A-D, 4A-D).

\section{The PKKS Receptors: A Potential Role of Bradykinin Receptors in Liver Fibrosis}

Finally, we assessed the receptors of the PKKS. F2r mRNA expression (Figure 8A) increased significantly at the early and progressive stages of liver fibrosis, whereas the F2rl1 mRNA level (Figure 8B) that was unchanged in acute and after 2.5-week liver injury increased at the 4 th, 6 th, and 7 th weeks of $\mathrm{CCl}_{4}$ treatment, suggesting a delayed induction. The F2r gene (Figure $8 \mathrm{C}$, Supplementary Figures 3A-D, 4A-D) exhibited more relationship at the 4th and 7th weeks, while the F2rl1 gene (Figure 8D, Supplementary Figures 3A-D, 4A-D) showed positive correlation with the Lgals 3 and $\mathrm{Ccn} 2$ genes, and the fibrotic area across 4- and 7-week time points. Compared to the Klkb1 gene, the F2r and F2rl1 genes were strongly associated to the myofibroblast activators and fibrotic areas, thus confirming the involvement of other ligands. An increase in the Bdkrb2 gene expression over all time points was observed while the Bdkrb1 gene expression peaked at the 4 th week (Figures 8E,F). Analysis by correlation showed a strong relationship of the Bdrkb2 gene with the myofibroblast activation markers and the fibrotic area in the early and progressive stages of liver fibrosis, thereby strengthening the relationship of the Kngl gene to liver fibrosis and conferring a possible similar pattern of regulation of the Bdkrb2 and Kng1 genes (Figure 8G; Supplementary Figures 3A-D, 4A-D).

\section{DISCUSSION}

Many studies have investigated mechanisms driving hepatic injury and fibrosis, extracellular matrix remodeling, and regeneration pattern. These processes implicated different patterns of coagulation, cell death, inflammation, and myofibroblast activation. Yet, the connection among these patterns is incomplete as more are being implicated in the 
pathogenesis of liver injury and fibrosis. In this study, we examined the gene expression profile of the components of the PKKS in liver injury and fibrosis and associated their expression levels to pathways that influence liver disease such as cell death, inflammation, and fibrosis.

One important finding of this study is the significant expression of Klkb1, Kng1, and F2r mRNA levels in acute liver injury, which was positively associated with the increase in cell death marker, Hmgb1 gene expression, as well as proinflammatory and immune-recruiting markers such as Illb, Ccl3, Adgre1, Ly6c, and neutrophil activation gene expression, Mpo. In addition, our data provided the first observation of the proliferation of hepatic HepG2 cells in response to plasma kallikrein, and the concomitant induction of inflammatory cytokine, IL1B, and myofibroblast activator genes CCN2 and LGALS3 in HepG2 cells, respectively. Last, the gene expression of Bdkrb2 was significantly induced in response to chronic liver injury in C57BL6/J mice and was positively correlated with the progression of liver fibrosis.

Few studies have addressed the role of PKKS in liver injury. Earlier studies by Borges et al. showed the increased clearance rate of plasma kallikrein by the exsanguinated liver in the murine model of acute inflammation (Martins et al., 1992), while it decreased in chronic liver injury (Toledo et al., 1995). Further investigation implicated a galectin-mediated pathway in endocytic clearance of the plasma kallikrein protein in the liver (Nagaoka et al., 2003). In this regard, our study showed that plasma kallikrein treatment stimulated the LGALS3 gene expression in hepatocytes. Intra- and extracellular galectin-3 play a role in the regulation of phagocytosis-induced activation of hepatic stellate cells (Jiang et al., 2012). Our study points to the upregulation of the mRNA expression of Lgals-3 at day 1 of the acute liver injury model and chronic liver injury, and suggests a potential role in liver injury. Furthermore, the temporal increase in the gene expressions of Klkb1 and F2r in acute liver injury may suggest a potential functional link between them. In addition, our data showed that plasma kallikrein treatment induced the gene expression of F2r and Illb, and a concomitant increase in the proliferation of both untreated and $1 \mathrm{mM} \mathrm{CCl}_{4}$-treated HepG2 cells. The inability of plasma kallikrein to induce an increase in the proliferation of $2 \mathrm{mM} \mathrm{CCl}_{4}$-treated HepG2 cells could be attributed to the decreased viability limiting the proliferation of these cells. Other studies have shown that plasma kallikrein can promote the proliferation in synovial cells (Dai et al., 2012). In this regard, our data pointed to a strong association between the Klkb1 and Hmgb1 gene expressions. Chen et al. (2014) showed that HMGB1 acts as a driver of acute liver injury (Chen et al., 2014) and inflammation. The inflammatory roles of HMGB1 (El Gazzar, 2007) could potentially modulate the expressions of the PKKS as indicated by their positive association.

Several studies have implicated the infiltration of immune cells as drivers of liver injury (Weiskirchen et al., 2018; Dong, 2019; Zhangdi et al., 2019). The PKKS is involved in the recruitment of immune cells (Gobel et al., 2019), and our study revealed their positive association to gene expression of recruiting immune cells. The relationship between the PKKS and neutrophils as indicated by the Ly6g and Mpo gene expressions depicts an activated phenotype which could be involved in acute liver injury progression or resolution. The PKKS especially through plasma kallikrein and bradykinin contributes to neutrophil activation and thereby inflammation (Kenne et al., 2019). This activation and inflammation as previously described require the liver sinusoidal endothelial cells in propagating or resolving liver injury or fibrosis (Kenne et al., 2019; McDonald and Kubes, 2012). Interestingly, the clustering of plasma kallikrein around injured areas as seen by immunohistochemistry could be attributed to its release from immune cells, eventually promoting hepatocyte proliferation. Our result is the first to describe the cluster formation of plasma kallikrein around necrotic areas and the possible proliferation of hepatocytes by plasma kallikrein.

In our chronic liver injury model, which mimics liver fibrosis time points of fibrogenesis and fibrosis, a different pattern of the PKKS gene expression was observed compared to that of acute liver injury. The Klkb1 mRNA level showed a decreased expression over all time points with no association to the myofibroblast activators and collagen deposition. Yet, in liver inflammation and fibrosis, plasma kallikrein cleaves the transforming growth factor, beta 1 (Kuniharu et al., 2002; Hara et al., 2014). This observation and our study suggest a feedback regulation over the Klkb1 gene. Nevertheless, receptors for plasma kallikrein, F2r, and F2rl1, mRNA expression, were increased at all time points, except at 2.5 weeks. This was expected as they are upregulated along with agonists, thrombin, factor Xa, and mast tryptase (Borensztajn et al., 2008; Borensztajn et al., 2010; Knight et al., 2012; Nault et al., 2016; Rullier et al., 2008). PAR1 induces the upregulation of CCN2 (Rullier et al., 2008). Our study highlights that the regulation of F2r could have affected in part the Ccn2 gene expression. Also, while F2r gene expression showed a strong positive association at 4 and 7 weeks of fibrosis establishment, the F2rl1 gene expression delayed fibrosis to 4 weeks through 7 weeks. Since both genes are significantly associated to the myofibroblast activator, CCN2, this supports previous experiments of PAR1 as an inducer of CCN2. Although the total knockout of F2r gene did not completely abrogate the expression of the Ccn2 gene (Rullier et al., 2008), compensatory PAR-like players such as PAR3 and PAR4 may be involved. Also, it is established that a cross talk exists between PAR1 and PAR2, especially with the transforming growth factor, beta 1 system (Ungefroren et al., 2018). Here we described that the F2rl1 mRNA expression showed more association to fibrosis, especially at 6and 7-week time points. This is also prominent with the Lgals3 mRNA expression at 4, 6, and 7 weeks. Our study reveals a potential relationship between $\mathrm{F} 2 \mathrm{rl1}$ and myofibroblast activators, Lgals3 and Ccn2, and the fibrotic area in early, progressive, and late fibrosis.

Furthermore, the Kngl gene expression showed a strong negative association to mRNA levels of Lgals3, Ccn2, and fibrotic area, except at the 6-week time point, corresponding to the relationship observed in acute liver injury. Kng1 gene expression is likely to be linked to liver fibrosis as supported by studies showing the inhibition of thrombin-induced platelet aggregation by bradykinin in humans (Murphey et al., 2006). Likewise, Sancho-Bru et al. (2007) showed that the infusion of 


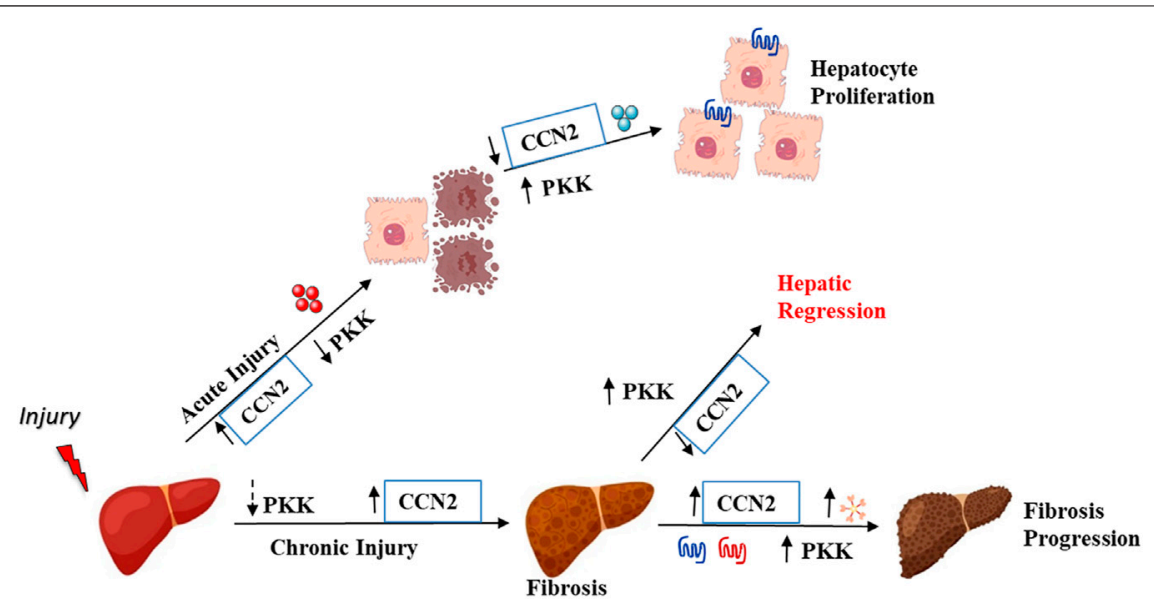

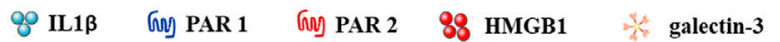

FIGURE 9 | Plasma kallikrein-kinin system diverse roles in liver injury.

bradykinin in extracted rat hepatocytes corrected hepatocellular damage in a chronic liver injury model (Sancho-Bru et al., 2007). However, in another experimental model of trichloroethylene induction of liver injury, bradykinin activated the Kupffer cells through BDKRB2 and contributed to liver injury (Zhang et al., 2019). This shows the varying interactions of the PKKS with various cell types within the liver architecture. However, our data showed that bradykinin receptors, especially Bdkrb2 mRNA level, are positively associated with liver fibrosis, implicating a potential role for these receptors in liver injury.

Overall, our findings implicated the involvement of plasma kallikrein in the hepatic milieu by stimulating LGALS3, CCN2, and IL1B, and suggest a regulation of the increase in proliferation of hepatocytes-a factor needed in repopulating hepatocytes in liver regeneration (Figure 9). Although these findings were done on cultured cells, our in vivo study showed the presence of plasma kallikrein in clusters around necrotic areas of the injured liver. This indicates its possible role in either protective or wound resolution processes. We are currently conducting studies exploring the function of plasma kallikrein in the liver, in terms of cause and effect, knowing fully well that an injured liver impairs many of its hepatic roles. Our future investigation is to relate plasma kallikrein to important functions of the liver such as metabolism, detoxification, inflammation, immunity, and blood coagulation. This will involve pharmacological inhibition or invalidation of the plasma kallikrein gene. In the present study, we also identified new relationships and interactions among the following: Kng1, F2r, F2rl1, Bdkrb1, and Bdkrb2 mRNA expression to Ccn2 and Lgals3 mRNA levels, and fibrosis in relation to chronic liver injury. This development among Kng1, Bdkrb1, and Bdkrb2 gene expressions, and myofibroblast activators and chronic liver injury creates a new direction in the study of liver fibrosis and its resolution. It is important to define the functional role of these genes in the development and progression of liver injury in order to identify new targets for intervention.

\section{DATA AVAILABILITY STATEMENT}

The original contributions presented in the study are included in the article/Supplementary Material; further inquiries can be directed to the corresponding author.

\section{ETHICS STATEMENT}

The animal study was reviewed and approved by the American University of Beirut, IACUC approvals 19-08-541 and 19-08-542.

\section{AUTHOR CONTRIBUTIONS}

Conceptualization of the research was performed by $\mathrm{EH}, \mathrm{AH}, \mathrm{AJ}$, and IA; methodology by IA, MJ, MM, DH, RAS, GEA, and MK; data curation, IA and $\mathrm{MJ}$; formal analysis, IA and MJ; writing-original draft preparation, IA; writing-review and editing, IA, AH, AJ, MM, MK, DH, RAS, GEA, MJ, and EH; funding acquisition, $\mathrm{EH}, \mathrm{AH}$, and $\mathrm{AJ}$. All authors have read and approved the publication of this article.

\section{FUNDING}

This work was funded by grants from the Medical Practice Plan (MPP), Faculty of Medicine, AUBMC (AH and AJ) and jointly funded with the support of the National Council for Scientific 
Research in Lebanon (CNRS-L) and the Lebanese University (\#01-10-17; AH and EH).

\section{ACKNOWLEDGMENTS}

The authors thank Julnar Usta (American University of Beirut) for generously providing the hepatocellular carcinoma cells (HepG2). IA and DH thank the Mastercard Foundation for

\section{REFERENCES}

Abdallah, R. T., Keum, J.-S., Lee, M.-H., Wang, B., Gooz, M., Luttrell, D. K., et al. (2010). Plasma Kallikrein Promotes Epidermal Growth Factor Receptor Transactivation and Signaling in Vascular Smooth Muscle through Direct Activation of Protease-Activated Receptors. J. Biol. Chem. 285 (45), 35206-35215. doi:10.1074/jbc.M110.171769

Akita, K., Okuno, M., Enya, M., Imai, S., Moriwaki, H., Kawada, N., et al. (2002). Impaired Liver Regeneration in Mice by Lipopolysaccharide via TNF-A/ kallikrein-Mediated Activation of Latent TGF- $\beta$. Gastroenterology 123 (1), 352-364. doi:10.1053/gast.2002.34234

Borensztajn, K., Stiekema, J., Nijmeijer, S., Reitsma, P. H., Peppelenbosch, M. P., and Spek, C. A. (2008). Factor Xa Stimulates Proinflammatory and Profibrotic Responses in Fibroblasts via Protease-Activated Receptor-2 Activation. Am. J. Pathol. 172 (2), 309-320. doi:10.2353/ajpath.2008.070347

Borensztajn, K., Von Der Thüsen, J. H., Peppelenbosch, M. P., and Spek, C. A. (2010). The Coagulation Factor Xa/protease Activated Receptor-2 axis in the Progression of Liver Fibrosis: a Multifaceted Paradigm. J. Cel Mol Med 14 (1-2), 143-153. doi:10.1111/j.1582-4934.2009.00980.x

Chen, M., Huang, W., Wang, C., Nie, H., Li, G., Sun, T., et al. (2014). High-mobility Group Box 1 Exacerbates CCl4-Induced Acute Liver Injury in Mice. Clin. Immunol. 153 (1), 56-63. doi:10.1016/j.clim.2014.03.021

Dai, J., Agelan, A., Yang, A., Zuluaga, V., Sexton, D., Colman, R. W., et al. (2012). Role of Plasma Kallikrein-Kinin System Activation in Synovial Recruitment of Endothelial Progenitor Cells in Experimental Arthritis. Arthritis Rheum. 64 (11), 3574-3582. doi:10.1002/art.34607

Dong, X., Liu, J., Xu, Y., and Cao, H. (2019). Role of Macrophages in Experimental Liver Injury and Repair in Mice (Review). Exp. Ther. Med. 17 (5), 3835-3847. doi:10.3892/etm.2019.7450

El Gazzar, M. (2007). HMGB1 Modulates Inflammatory Responses in LPSActivated Macrophages. Inflamm. Res. 56 (4), 162-167. doi:10.1007/s00011006-6112-0

Endig, J., Unrau, L., Sprezyna, P., Rading, S., Karsak, M., Goltz, D., et al. (2019). Acute Liver Injury after CCl4 Administration Is Independent of Smad7 Expression in Myeloid Cells. Int. J. Mol. Sci. 20 (22), 5528. doi:10.3390/ ijms20225528

Gao, B., and Bataller, R. (2011). Alcoholic Liver Disease: Pathogenesis and New Therapeutic Targets. Gastroenterology 141 (5), 1572-1585. doi:10.1053/ j.gastro.2011.09.002

Gieseler, F., Ungefroren, H., Settmacher, U., Hollenberg, M. D., and Kaufmann, R. (2013). Proteinase-activated Receptors (PARs) - Focus on Receptor-ReceptorInteractions and Their Physiological and Pathophysiological Impact. Cell Commun. Signaling 11 (86), 86-26. doi:10.1186/1478-811X-11-86

Göbel, K., Asaridou, C.-M., Merker, M., Eichler, S., Herrmann, A. M., Geuß, E., et al. (2019). Plasma Kallikrein Modulates Immune Cell Trafficking during Neuroinflammation via PAR2 and Bradykinin Release. Proc. Natl. Acad. Sci. USA 116 (1), 271-276. doi:10.1073/pnas.1810020116

Habib, A., Chokr, D., Wan, J., Hegde, P., Mabire, M., Siebert, M., et al. (2019). Inhibition of Monoacylglycerol Lipase, an Anti-inflammatory and Antifibrogenic Strategy in the Liver. Gut 68 (3), 522-532. doi:10.1136/ gutjnl-2018-316137

Hara, M., Kirita, A., Kondo, W., Matsuura, T., Nagatsuma, K., Dohmae, N., et al. (2014). LAP Degradation Product Reflects Plasma Kallikrein-dependent TGF- $\beta$ their sponsorship, training, and support through their graduate education at the American University of Beirut.

\section{SUPPLEMENTARY MATERIAL}

The Supplementary Material for this article can be found online at: https://www.frontiersin.org/articles/10.3389/fphar.2021.715111/ full\#supplementary-material

Activation in Patients with Hepatic Fibrosis. SpringerPlus 3 (221), 1-11. doi:10.1186/2193-1801-3-221

Heuberger, D. M., and Schuepbach, R. A. (2019). Protease-activated Receptors (PARs): Mechanisms of Action and Potential Therapeutic Modulators in PARDriven Inflammatory Diseases. Thromb. J 17, 4. doi:10.1186/s12959-0190194-8

Horvatits, T., Drolz, A., Trauner, M., and Fuhrmann, V. (2019). Liver Injury and Failure in Critical Illness. Hepatology 70 (6), 2204-2215. doi:10.1002/hep.30824

Jiang, J. X., Chen, X., Hsu, D. K., Baghy, K., Serizawa, N., Scott, F., et al. (2012) Galectin-3 Modulates Phagocytosis-Induced Stellate Cell Activation and Liver Fibrosis In Vivo. Am. J. Physiology-Gastrointestinal Liver Physiol. 302 (4), G439-G446. doi:10.1152/ajpgi.00257.2011

Kaplan, A. P., and Ghebrehiwet, B. (2010). The Plasma Bradykinin-Forming Pathways and its Interrelationships with Complement. Mol. Imm., 47(13), 2161-2169. doi:10.1016/j.molimm.2010.05.010

Joseph, K., Tholanikunnel, B. G., and Kaplan, A. P. (2009). Factor XII-independent Cleavage of High-Molecular-Weight Kininogen by Prekallikrein and Inhibition by C1 Inhibitor. J. Allergy Clin. Immunol. 124 (1), 143-149. doi:10.1016/ j.jaci.2009.02.006

Kallis, Y. N., Scotton, C. J., Mackinnon, A. C., Goldin, R. D., Wright, N. A., Iredale, J. P., et al. (2014). Proteinase Activated Receptor 1 Mediated Fibrosis in a Mouse Model of Liver Injury: a Role for Bone Marrow Derived Macrophages. PLoS One 9 (1), e86241. doi:10.1371/journal.pone.0086241

Kenne, E., Rasmuson, J., Renné, T., Vieira, M. L., Müller-Esterl, W., Herwald, H., et al. (2019). Neutrophils Engage the Kallikrein-kinin System to Open up the Endothelial Barrier in Acute Inflammation. FASEB J. 33 (2), 2599-2609. doi:10.1096/fj.201801329R

Kitasato, L., Yamaoka-Tojo, M., Hashikata, T., Ishii, S., Kameda, R., Shimohama, T., et al. (2014). Factor Xa in Mouse Fibroblasts May Induce Fibrosis More Than Thrombin. Int. Heart J. 55 (4), 357-361. doi:10.1536/ihj.13-351

Knight, V., Tchongue, J., Lourensz, D., Tipping, P., and Sievert, W. (2012). Protease-activated Receptor 2 Promotes Experimental Liver Fibrosis in Mice and Activates Human Hepatic Stellate Cells. Hepatology 55 (3), 879-887. doi:10.1002/hep. 24784

Li, M., Qin, X.-Y., Furutani, Y., Inoue, I., Sekihara, S., Kagechika, H., et al. (2018). Prevention of Acute Liver Injury by Suppressing Plasma Kallikrein-dependent Activation of Latent TGF- $\beta$. Biochem. Biophysical Res. Commun. 504 (4), 857-864. doi:10.1016/j.bbrc.2018.09.026

Man, L. L., Liu, F., Wang, Y. J., Song, H. H., Xu, H. B., Zhu, Z. W., et al. (2015). The HMGB1 Signaling Pathway Activates the Inflammatory Response in Schwann Cells. Neural Regen. Res. 10 (10), 1706-1712. doi:10.4103/1673-5374.167773

Marra, F., Grandaliano, G., Valente, A. J., and Abboud, H. E. (1995). Thrombin Stimulates Proliferation of Liver Fat-Storing Cells and Expression of Monocyte Chemotactic Protein-1: Potential Role in Liver Injury*1. Hepatology 22, 780-787. doi:10.1016/0270-9139(95)90297-x

Martins, B., Kouyoumdjian, M., Limaos, E. A., and Borges, D. R. (1992). The Clearance Rate of Plasma Kallikrein by the Liver Increases during the Acutephase Response to Inflammation. Agents and Actions 37 (1-2), 111-113. doi:10.1007/BF01987898

McDonald, B., and Kubes, P. (2012). Neutrophils and Intravascular Immunity in the Liver during Infection and Sterile Inflammation. Toxicol. Pathol. 40 (2), 157-165. doi:10.1177/0192623311427570

Mitchell, C., Couton, D., Couty, J.-P., Anson, M., Crain, A.-M., Bizet, V., et al. (2009). Dual Role of CCR2 in the Constitution and the Resolution of Liver 
Fibrosis in Mice. Am. J. Pathol. 174 (5), 1766-1775. doi:10.2353/ ajpath.2009.080632

Moreau, M. E., Garbacki, N., Molinaro, G., Brown, N. J., Marceau, F., and Adam, A. (2005). The Kallikrein-Kinin System: Current and Future Pharmacological Targets. J. Pharmacol. Sci. 99 (1), 6-38. doi:10.1254/jphs.srj05001x

Mossanen, J. C., Krenkel, O., Ergen, C., Govaere, O., Liepelt, A., Puengel, T., et al. (2016). Chemokine (C-C Motif) Receptor 2-positive Monocytes Aggravate the Early Phase of Acetaminophen-Induced Acute Liver Injury. Hepatology 64 (5), 1667-1682. doi:10.1002/hep.28682

Murphey, L. J., Malave, H. A., Petro, J., Biaggioni, I., Byrne, D. W., Vaughan, D. E., et al. (2006). Bradykinin and its Metabolite Bradykinin 1-5 Inhibit ThrombinInduced Platelet Aggregation in Humans. J. Pharmacol. Exp. Ther. 318 (3), 1287-1292. doi:10.1124/jpet.106.104026

Nagaoka, M. R., Kouyoumdjian, M., and Borges, D. R. (2003). Hepatic Clearance of Tissue-type Plasminogen Activator and Plasma Kallikrein in Experimental Liver Fibrosis. Liver Int. 23 (6), 476-483. doi:10.1111/j.1478-3231.2003.00872.x

Nault, R., Fader, K. A., Kopec, A. K., Harkema, J. R., Zacharewski, T. R., and Luyendyk, J. P. (2016). From the Cover: Coagulation-Driven Hepatic Fibrosis Requires Protease Activated Receptor-1 (PAR-1) in a Mouse Model of TCDDElicited Steatohepatitis. Toxicol. Sci. 154 (2), 381-391. doi:10.1093/toxsci/ kfw175

Pant, A., Kopec, A. K., and Luyendyk, J. P. (2018). Role of the Blood Coagulation cascade in Hepatic Fibrosis. Am. J. Physiology-Gastrointestinal Liver Physiol. 315 (2), G171-G176. doi:10.1152/ajpgi.00402.2017

Paudel, Y. N., Shaikh, M. F., Chakraborti, A., Kumari, Y., Aledo-Serrano, Á., Aleksovska, K., et al. (2018). HMGB1: A Common Biomarker and Potential Target for TBI, Neuroinflammation, Epilepsy, and Cognitive Dysfunction. Front. Neurosci. 12, 628. doi:10.3389/fnins.2018.00628

Real, M., Barnhill, M. S., Higley, C., Rosenberg, J., and Lewis, J. H. (2019). DrugInduced Liver Injury: Highlights of the Recent Literature. Drug Saf. 42 (3), 365-387. doi:10.1007/s40264-018-0743-2

Reichel, C. A., Puhr-Westerheide, D., Zuchtriegel, G., Uhl, B., Berberich, N., Zahler, S., et al. (2012). C-C Motif Chemokine CCL3 and Canonical Neutrophil Attractants Promote Neutrophil Extravasation through Common and Distinct Mechanisms. Blood 120 (4), 880-890. doi:10.1182/blood-2012-01402164

Ribeiro, M. S., Dellalibera-Joviliano, R., Becari, C., Teixeira, F. R., Araujo, P. V., Piccinato, C. E., et al. (2014). Characterization of the Kallikrein-Kinin System, Metalloproteinases, and Their Tissue Inhibitors in the In-Stent Restenosis after Peripheral Percutaneous Angioplasty. Ann. Vasc. Surg. 28 (4), 1005-1015. doi:10.1016/j.avsg.2013.11.014

Rullier, A., Gillibert-Duplantier, J., Costet, P., Cubel, G., Haurie, V., Petibois, C., et al. (2008). Protease-activated Receptor 1 Knockout Reduces Experimentally Induced Liver Fibrosis. Am. J. Physiology-Gastrointestinal Liver Physiol. 294 (1), G226-G235. doi:10.1152/ajpgi.00444.2007

Sancho-Bru, P., Bataller, R., Fernandez-Varo, G., Moreno, M., Ramalho, L. N., Colmenero, J., et al. (2007). Bradykinin Attenuates Hepatocellular Damage and Fibrosis in Rats with Chronic Liver Injury. Gastroenterology 133 (6), 2019-2028. doi:10.1053/j.gastro.2007.09.023

Schmaier, A. H. (2016). The Contact Activation and Kallikrein/kinin Systems: Pathophysiologic and Physiologic Activities. J. Thromb. Haemost. 14 (1), 28-39. doi:10.1111/jth.13194

Shearer, A. M., Rana, R., Austin, K., Baleja, J. D., Nguyen, N., Bohm, A., et al. (2016). Targeting Liver Fibrosis with a Cell-Penetrating Protease-Activated
Receptor-2 (PAR2) Pepducin. J. Biol. Chem. 291 (44), 23188-23198. doi:10.1074/jbc.M116.732743

Singh, S., Allen, A. M., Wang, Z., Prokop, L. J., Murad, M. H., and Loomba, R. (2015). Fibrosis Progression in Nonalcoholic Fatty Liver vs Nonalcoholic Steatohepatitis: a Systematic Review and Meta-Analysis of Paired-Biopsy Studies. Clin. Gastroenterol. Hepatol. 13 (4), 643-654. doi:10.1016/ j.cgh.2014.04.014

Stravitz, R. T., and Lee, W. M. (2019). Acute Liver Failure. The Lancet 394 (10201), 869-881. doi:10.1016/s0140-6736(19)31894-x

Toledo, C. F., Kouyoumdjian, M., Lanzoni, V. P., and Borges, D. R. (1995). Plasma Kallikrein Clearance by the Liver of Chronic Carbon Tetrachloride-Treated Rats. J. Gastroenterol. Hepatol. 10 (2), 165-168. doi:10.1111/j.14401746.1995.tb01072.x

Ungefroren, H., Gieseler, F., Kaufmann, R., Settmacher, U., Lehnert, H., and Rauch, B. (2018). Signaling Crosstalk of TGF-B/alk5 and PAR2/PAR1: A Complex Regulatory Network Controlling Fibrosis and Cancer. Int. J. Mol. Sci. 19 (6), 1568. doi:10.3390/ijms19061568

Wang, K. (2014). Molecular Mechanisms of Liver Injury: Apoptosis or Necrosis. Exp. Toxicologic Pathol. 66 (8), 351-356. doi:10.1016/j.etp.2014.04.004

Weiskirchen, R., Weiskirchen, S., and Tacke, F. (2018). Recent Advances in Understanding Liver Fibrosis: Bridging Basic Science and Individualized Treatment Concepts. F1000Res 7, 921. doi:10.12688/f1000research.14841.1

Zhang, C., Dong, H., Chen, F., Wang, Y., Ma, J., and Wang, G. (2019a). The HMGB1RAGE/TLRTNFa Signaling Pathway May Contribute to Kidney Injury Induced by Hypoxia. Exp. Ther. Med. 17 (1), 17-26. doi:10.3892/etm.2018.6932

Zhang, J., Li, N., Yang, L., Xie, H., Yang, Y., Wang, H., et al. (2019b). Bradykinin Contributes to Immune Liver Injury via B2R Receptor-Mediated Pathways in Trichloroethylene Sensitized Mice: A Role in Kupffer Cell Activation. Toxicology 415, 37-48. doi:10.1016/j.tox.2019.01.015

Zhang, J., Li, N., Yang, L., Zang, D., Yang, P., Wang, H., et al. (2018). Role of Selective Blocking of Bradykinin B1 Receptor in Attenuating Immune Liver Injury in Trichloroethylene-Sensitized Mice. Cytokine 108, 71-81. doi:10.1016/ j.cyto.2018.03.024

Zhangdi, H.-J., Su, S.-B., Wang, F., Liang, Z.-Y., Yan, Y.-D., Qin, S.-Y., et al. (2019). Crosstalk Network Among Multiple Inflammatory Mediators in Liver Fibrosis. World. J. Gastroentrol. 25 (33), 4835-4849. doi:10.3748/wjg.v25.i33.4835

Conflict of Interest: The authors declare that the research was conducted in the absence of any commercial or financial relationships that could be construed as a potential conflict of interest.

Publisher's Note: All claims expressed in this article are solely those of the authors and do not necessarily represent those of their affiliated organizations, or those of the publisher, the editors, and the reviewers. Any product that may be evaluated in this article, or claim that may be made by its manufacturer, is not guaranteed or endorsed by the publisher.

Copyright (c) 2021 Ahmed, Jaffa, Moussa, Hatem, El-Achkar, Al Sayegh, Karam, Hamade, Habib and Jaffa. This is an open-access article distributed under the terms of the Creative Commons Attribution License (CC BY). The use, distribution or reproduction in other forums is permitted, provided the original author(s) and the copyright owner(s) are credited and that the original publication in this journal is cited, in accordance with accepted academic practice. No use, distribution or reproduction is permitted which does not comply with these terms. 